\author{
Alterstice \\ Revue internationale de la recherche interculturelle \\ International Journal of Intercultural Research \\ Revista International de la Investigacion Intercultural
}

\title{
Mouvement des frontières identitaires dans les dessins d'élèves immigrants
}

\section{Caroline Beauregard, Garine Papazian-Zohrabian et Cécile Rousseau}

Volume 7, numéro 2, 2017

URI : https://id.erudit.org/iderudit/1052573ar

DOI : https://doi.org/10.7202/1052573ar

Aller au sommaire du numéro

Éditeur(s)

Alterstice

ISSN

1923-919X (numérique)

Découvrir la revue

Citer cet article

Beauregard, C., Papazian-Zohrabian, G. \& Rousseau, C. (2017). Mouvement des frontières identitaires dans les dessins d'élèves immigrants. Alterstice, $7(2)$, 105-116. https://doi.org/10.7202/1052573ar

\section{Résumé de l'article}

Pour l'élève immigrant, le contact interculturel occasionné par l'immigration génère une prise de conscience des différences et des ressemblances entre lui et les autres, ce qui influence son sentiment d'ipséité et sa perception de l'altérité. C'est notamment en jouant et en déplaçant les limites de son identité que l'enfant (re)définit qui il est. Cependant, cette mobilité des frontières identitaires peut ne pas être facilement perceptible. Une manière de rendre ce mouvement plus concret est d'avoir recours au dessin. Par le dessin, l'élève peut dévoiler d'importantes informations sur lui-même dont il n'est pas nécessairement conscient. Le dessin peut également lui permettre d'utiliser des symboles qui facilitent l'expression d'expériences qui n'ont pas été extériorisées et de les partager avec autrui. Enfin, le dessin peut ouvrir l'accès aux identifications vécues par l'enfant en donnant une forme symbolique ou figurative aux personnes auxquelles il s'identifie ou non.

L'objectif de cet article est d'examiner les dessins créés par deux jeunes immigrants sous l'angle du mouvement des frontières définissant l'identité et l'altérité. L'étude s'appuie sur des données obtenues dans le cadre d'une recherche-intervention de 14 semaines dans des classes d'accueil pour enfants immigrants d'une école primaire multiethnique de Montréal (Canada). À partir d'entrevues semi-dirigées auprès d'élèves immigrants, de leurs parents et de leurs enseignants, ainsi qu'à partir d'autoportraits thématiques et de dessins libres commentés qu'ils ont réalisés lors de l'intervention, les auteures décrivent comment deux jeunes élèves nouvellement arrivés au Québec (re)définissent leur identité en jouant dans leurs dessins avec les limites identitaires. Les résultats suggèrent que les élèves immigrants ont recours à des stratégies d'assimilation et de différenciation qui sont illustrées dans les dessins qu'ils réalisent, de façon séparée ou simultanée. Le dessin semble soutenir la (re)définition de l'identité de ces enfants.
Droits d'auteur (c) Caroline Beauregard, Garine Papazian-Zohrabian et Cécile Rousseau, 2018
Ce document est protégé par la loi sur le droit d'auteur. L’utilisation des services d'Érudit (y compris la reproduction) est assujettie à sa politique d'utilisation que vous pouvez consulter en ligne.

https://apropos.erudit.org/fr/usagers/politique-dutilisation/ 


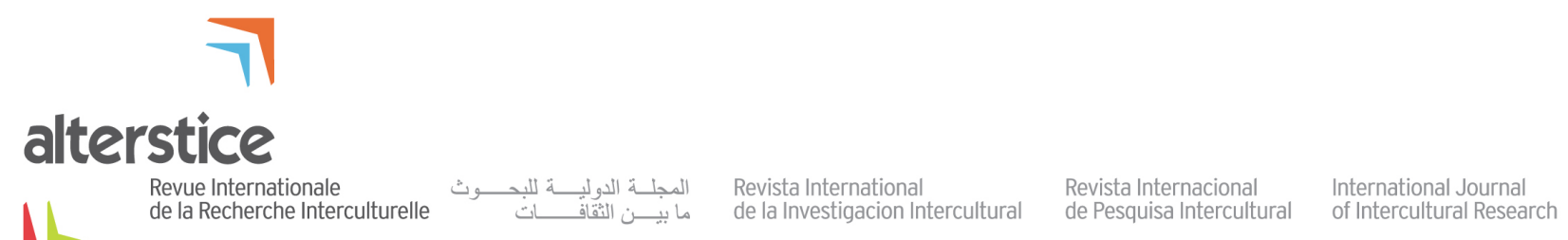

ARTICLE HORS THÈME

\title{
Mouvement des frontières identitaires dans les dessins d'élèves immigrants
}

\author{
Caroline Beauregard $^{1,2}$, Garine Papazian-Zohrabian ${ }^{2,3}$ et Cécile Rousseau ${ }^{2,4}$
}

\section{Résumé}

Pour l'élève immigrant, le contact interculturel occasionné par l'immigration génère une prise de conscience des différences et des ressemblances entre lui et les autres, ce qui influence son sentiment d'ipséité et sa perception de l'altérité. C'est notamment en jouant et en déplaçant les limites de son identité que l'enfant (re)définit qui il est. Cependant, cette mobilité des frontières identitaires peut ne pas être facilement perceptible. Une manière de rendre ce mouvement plus concret est d'avoir recours au dessin. Par le dessin, l'élève peut dévoiler d'importantes informations sur lui-même dont il n'est pas nécessairement conscient. Le dessin peut également lui permettre d'utiliser des symboles qui facilitent l'expression d'expériences qui n'ont pas été extériorisées et de les partager avec autrui. Enfin, le dessin peut ouvrir l'accès aux identifications vécues par l'enfant en donnant une forme symbolique ou figurative aux personnes auxquelles il s'identifie ou non.

L'objectif de cet article est d'examiner les dessins créés par deux jeunes immigrants sous l'angle du mouvement des frontières définissant l'identité et l'altérité. L'étude s'appuie sur des données obtenues dans le cadre d'une recherche-intervention de 14 semaines dans des classes d'accueil pour enfants immigrants d'une école primaire multiethnique de Montréal (Canada). À partir d'entrevues semi-dirigées auprès d'élèves immigrants, de leurs parents et de leurs enseignants, ainsi qu'à partir d'autoportraits thématiques et de dessins libres commentés qu'ils ont réalisés lors de l'intervention, les auteures décrivent comment deux jeunes élèves nouvellement arrivés au Québec (re)définissent leur identité en jouant dans leurs dessins avec les limites identitaires. Les résultats suggèrent que les élèves immigrants ont recours à des stratégies d'assimilation et de différenciation qui sont illustrées dans les dessins qu'ils réalisent, de façon séparée ou simultanée. Le dessin semble soutenir la (re)définition de l'identité de ces enfants.

\section{Rattachement des auteures}

${ }^{1}$ Université du Québec en Abitibi-Témiscamingue, Canada; ${ }^{2}$ Centre de recherche Sherpa, du CIUSSS du Centreouest-de-I'île-de-Montréal; ${ }^{3}$ Université de Montréal, Montréal, Canada; ${ }^{4}$ Université McGill, Montréal, Canada

\section{Correspondance}

caroline.beauregard@uqat.ca

\section{Mots clés}

frontières identitaires, dessins, enfants immigrants, étrangeté, similarité

\section{Pour citer cet article}

Beauregard, C., Papazian-Zohrabian, G. et Rousseau, C. (2017). Mouvement des frontières identitaires dans les dessins d'élèves immigrants. Alterstice, $7(2), 105-116$. 


\section{Contexte}

Le Québec est l'une des provinces canadiennes recevant le plus grand nombre d'immigrants. En 2014, sur les 260404 immigrants et réfugiés reçus par le Canada, le Québec en a accueilli 50 294, se plaçant ainsi au $2^{\text {e rang }}$ après l'Ontario (Gouvernement du Canada, 2015). Plus du trois quarts de ces immigrants s'établissent dans la région métropolitaine de Montréal et plus de $20 \%$ d'entre eux ont moins de 15 ans (Gouvernement du Québec, 2015). Selon la loi sur l'instruction publique au Québec, les enfants immigrants ont l'obligation de fréquenter l'école francophone (Gouvernement du Québec, 1988). À Montréal, où près du quart des élèves (22,1\%) sont nés à l'étranger et où plus de $40 \%$ ont une langue maternelle autre que le français (Sévigny, 2016), il est généralement possible de mettre en place des mesures de francisation en classe d'accueil fermée, où les enfants immigrants sont regroupés dans les mêmes classes afin de suivre tous leurs cours (Armand, 2012; De Koninck et Armand, 2011). Ces classes d'accueil, ou d'intégration, ont pour mandat de franciser les enfants nouvellement arrivés au Québec, mais elles ont aussi pour mandat de favoriser leur adaptation psychosociale et scolaire (Ministère de l'Éducation, du Loisir et du Sport du Québec, 2014).

Considérant qu'une identité harmonieuse favorise l'adaptation psychosociale et la réussite scolaire des jeunes élèves (Faircloth, 2012; Phinney et Ong, 2007; Sinai, Kaplan, et Flum, 2012), le milieu scolaire doit donc porter une attention particulière à la manière dont les élèves construisent et expriment leur(s) identité(s), et ce, particulièrement en classe d'accueil. L'intervention par l'art propose une façon novatrice d'étudier l'expression identitaire d'élèves immigrants en abordant la question sous un nouvel angle. En effet, l'identité est généralement étudiée à l'aide de questionnaires ou d'entretiens. Toutefois, l'utilisation de ce type d'instruments plus structurés comporte le risque d’orienter les réponses données par l'élève (Marc, 2005). Ces instruments requièrent aussi la maîtrise de la langue parlée et écrite, ce qui pose certaines questions sur la validité des réponses obtenues de l'élève immigrant. Un outil basé sur l'expression non verbale, comme le dessin, serait ainsi beaucoup plus adéquat à la réalité de l'élève immigrant (Anzieu, Barbey, Bernard-Nez et Daymas, 2012). Ce texte propose donc d'examiner comment deux jeunes élèves immigrants expriment leur identité dans des dessins libres réalisés dans le cadre d'ateliers d'expression créatrice intitulés Art et Contes et offerts dans leur classe d'accueil. II a pour objectif d'interroger leurs perceptions et leur expression de l'altérité autour de leur expérience d'immigration, sous l'angle de la représentation graphique de ce qui les rend à la fois semblables et différents des autres. À ce sujet, les concepts d'ipséité et d'altérité peuvent éclairer la compréhension du phénomène identitaire lors de la migration. Ils sont présentés dans la section suivante.

\section{Immigration et identité : quand l'ipséité et l'altérité s'intensifient}

L'identité se décline en plusieurs dimensions (personnelle, sociale, nationale, etc.) et de nombreuses définitions existent, provenant de disciplines diverses telles que la psychologie, la psychanalyse, la sociologie ou I'anthropologie (Devereux, 1985; Frie, 2011; Vinsonneau, 2002). La définition retenue dans le cadre de ce texte est celle de l'identité personnelle du psychosociologue Pierre Tap qui souligne que :

mon identité est ce qui me rend semblable à moi-même et différent des autres, ce par quoi je me sens exister en tant que personne et en tant que personnage social (rôles, fonctions et relations), ce par quoi je me définis et me reconnais, me sens accepté et reconnu, ou rejeté et méconnu par autrui, par mes groupes ou ma culture d'appartenance. (1988, p. 69)

Ainsi, l'identité réfère à la fois à l'unicité de la personne et à sa ressemblance avec d'autres ce qui, à première vue, peut sembler illogique. Afin d'éclairer ce paradoxe, il est possible de s'appuyer sur deux concepts, celui de l'ipséité et celui de l'altérité. En effet, l'unicité d'un individu peut se rapprocher de son sentiment d'ipséité, puisque l'ipséité se rapporte à l'aspect de l'identité qui est maintenue dans le temps en dépit du changement (Ricœur, 1990). Malgré les expériences vécues par une personne, il y aura toujours certains éléments qui permettront de la reconnaître et de ne pas la confondre avec quelqu'un d'autre. Quant à l'altérité, elle existe en opposition à l'identité, puisqu'elle implique la différence, une comparaison à soi (Jodelet, 2008). Quoique ces concepts puissent paraître distincts, ils sont néanmoins interdépendants. En examinant l'œuvre de Ricœur, Dosse (2006) souligne effectivement que "l'ipséité ne se construit pas dans un rapport analogique d'extériorité à l'autre, mais dans une implication, une véritable intrication à l'autre » (p. 663). Ainsi, c'est en entrant en contact avec l'autre, en se mêlant 
avec l'altérité, que se construit l'unicité des individus, leur identité. Autrement dit, c'est en se positionnant face à l'autre qui est différent qu'il est possible de se définir sur le plan identitaire (Augé, 1994).

Lors de la migration, l'ipséité et l'altérité s'intensifient. En effet, l'immigration provoque nécessairement une situation de contact avec l'altérité, où l'immigrant est confronté à la nécessité de se redéfinir comme un étranger dans la société d'accueil tout en étant différent de ceux restés au pays d'origine (Fouquet, 2007). Le contexte migratoire représente donc un cadre propice à un remaniement intense de l'identité. Dans le pays d'accueil, le contact avec d'autres groupes culturels génère une prise de conscience de la différence entre soi et autrui, que l'immigrant gérera de différentes façons en déplaçant les limites de son identité. Selon Barth (1995), les frontières de l'identité sont fluides et se déplacent en fonction de la distance que l'individu perçoit entre lui et les autres (la différence engendrant une plus grande distance et la ressemblance, une moins grande). Ce serait ce déplacement des frontières identitaires qui entraînerait une redéfinition des identités individuelles et collectives des individus et des groupes en contact.

Dans le contexte d'une classe d'accueil fréquentée par des enfants immigrants d'origines diverses, il est possible que les limites identitaires se déplacent davantage en raison de la présence d'un grand nombre de similitudes et de différences à l'intérieur de la classe. Dans ce contexte, l'élève immigrant doit se positionner vis-à-vis des autres et, pour ce faire, il activera certaines options identitaires. Celles-ci correspondent à la possibilité ou non d'appartenir à un groupe donné selon le partage ou non de significations et de symboles communs (Song, 2003; Vinsonneau, 2012). Ces options identitaires permettent l'inclusion ou l'exclusion de certaines personnes d'un groupe donné, que cette exclusion soit volontaire ou non. Lorsque l'élève immigrant choisit d'activer ou non certaines options identitaires, il fait preuve de stratégies identitaires (Camilleri, Kastersztein, Lipiansky, Malewska-Peyre, TaboadaLeonetti et Vasquez., 1990). En optant pour certains comportements, attitudes ou mécanismes, l'élève met en valeur les aspects de son identité qu'il croit conformes à la situation vécue et dissimule les aspects qui sont perçus négativement (Devereux, La Barre et Sinaceur, 2012; Vinsonneau, 2002, 2012). Ces stratégies identitaires sont de deux ordres. L'enfant fait appel à des stratégies d'assimilation lorsqu'il cherche à se rendre semblable à l'autre (l'enseignant ou ses pairs, par exemple) et à évacuer ce qui est différent, et il a plutôt recours à des stratégies de différenciation lorsqu'il fait ressortir son unicité et sa différence par rapport aux autres (Marc, 2005). Ce sont ces différentes stratégies qui conduisent à un mouvement des frontières identitaires et qui permettent à l'enfant immigrant de se (re)définir sur le plan identitaire.

\section{Le dessin et l'expression identitaire}

La nature des frontières identitaires fait que leur mouvement est difficilement tangible. Une façon de donner une forme matérielle à cette mobilité identitaire est d'avoir recours au dessin. Le dessin permet de manifester son monde intérieur, d'exprimer son identité et d'en recevoir le reflet. Par le processus de projection, l'élève révèle dans son dessin des informations sur lui-même dont il n'est pas nécessairement conscient ou dont il ne possède pas les capacités linguistiques lui permettant de les partager oralement (Anzieu et coll., 2012). Parmi ces informations figurent les identifications, qui concourent à la construction identitaire. En effet, par une succession d'identifications, l'enfant assimile certaines caractéristiques d'autrui et les transforme de façon complète ou partielle pour les intégrer à qui il est (Pontalis et Laplanche, 2004). En dessinant, l'enfant explore différentes identifications aux personnes qu'il côtoie et transforme ainsi son identité (Hawkins, 2002). Toutefois, les identifications sont rarement exprimées de façon directe par l'enfant, qui a recours à des symboles pour représenter une expérience ou une émotion qui n’a pas été extériorisée auparavant, ce qui lui permet alors de la partager et d'entrer en relation avec autrui (Stepakoff, 2007).

\section{Méthodologie}

\section{Intervention Art et Contes}

Le programme d'expression créatrice Art et Contes existe depuis les années 1990 et a été élaboré par l'équipe de recherche et d'intervention transculturelles (Erit) du centre de recherche Sherpa en collaboration avec le milieu scolaire et communautaire québécois (Erit, 2010). Il s'agit d'une série de 12 ateliers hebdomadaires d'une heure animés par deux intervenants externes (art-thérapeute et intervenant en théâtre) conjointement avec l'enseignant. 
Au début de chaque atelier, les enfants sont invités à former un cercle et à participer à un jeu (ex. faire un son et un mouvement que les autres reproduisent), afin d'activer l'énergie ludique et de créer une ambiance de détente et de plaisir. Suit une période d'histoires qui peuvent être racontées par les adultes, imaginées par les enfants lors de périodes de visualisation guidée ou encore racontées par les enfants. Par la suite, ces derniers sont invités à se regrouper par groupes de 4 ou 5 afin de créer un dessin libre individuel au pastel gras, sur une feuille A3 (30,5 par $43,7 \mathrm{~cm}$ ). Les consignes sont simples: ils peuvent dessiner ce qu'ils veulent, cela peut être relié à l'histoire entendue ou non. Afin de clore chaque atelier, les enfants reviennent en cercle et déposent, dans une boîte imaginaire, des éléments qu'ils ont aimés ou moins aimés de l'atelier.

\section{Collecte de données}

Dans le cadre de cette recherche-intervention de 14 semaines, le programme Art et Contes a été offert à deux classes d'accueil pour enfants immigrants d'une école primaire multiethnique de Montréal (Canada). Les enfants étaient âgés de 8 à 10 ans pour l'une des classes ( ${ }^{\mathrm{e}}$ cycle) et de 10 à 12 ans pour l'autre groupe ( $3^{\mathrm{e}}$ cycle). Dans les deux classes, tous les élèves ont pris part au programme d'intervention et 29 sur 31 ont participé à la recherche. Au total, 478 dessins libres commentés ont été recueillis. Une rencontre "préintervention " et une rencontre "postintervention" se sont ajoutées aux 12 ateliers, durant lesquelles les enfants étaient invités à dessiner leur autoportrait en deux temps: Moi, de l'extérieur (tel que je pense que les autres me voient) et Moi de l'intérieur (tel que je me perçois), et 116 autoportraits dirigés ont été recueillis dans ce cadre. Tout au long des 14 semaines, la chercheure-intervenante a tenu un journal de bord et compilé des notes de terrain en lien avec son observation participante lors des ateliers à titre d'intervenante.

À la fin de l'intervention, 4 enfants ont été sélectionnés (2 par classe) afin de participer à une entrevue semi-dirigée avec leur(s) parent(s) et une interprète. Les enfants ont été choisis en fonction de leur aisance avec la chercheureintervenante et de la richesse graphique de leurs dessins relativement au thème de l'expression identitaire. Avec l'enfant, l'entrevue consistait en une revue des dessins réalisés pendant Art et Contes, afin d'approfondir les commentaires de l'enfant sur chacun de ses dessins. Elle a également permis de recueillir des informations sur les thèmes de l'école, des amis et de la famille. Avec les parents, la discussion a porté sur l'histoire familiale et sur les thèmes de la langue, des croyances religieuses et des changements remarqués chez l'enfant depuis l'arrivée au Canada. Les enseignants des deux classes ont également été rencontrés afin de discuter de leur philosophie d'enseignement, des relations interpersonnelles dans leur classe et de la thématique de l'identité chez leurs élèves.

Ce texte s'appuie donc sur l'analyse des données en lien avec deux des enfants sélectionnés (dessins, entrevues et notes de terrain) : un garçon d'origine vénézuélienne et une jeune fille égyptienne, tous deux âgés de 11 ans et fréquentant la même classe. Ils ont été choisis pour illustrer cet article car le thème de l'étrangeté revenait régulièrement dans leurs dessins et pour les commentaires qu'ils ont faits. Le contenu et les thématiques de leurs dessins et de leur entrevue ont été mis en parallèle afin de relever les similitudes et les différences et de faire ressortir certains thèmes. Nous allons voir comment ces deux jeunes, nouvellement arrivés au Québec, (re)définissent leur(s) identité(s) en termes d'étrangeté et de similarité à l'autre et jouent avec les limites de leur(s) identité(s) dans leurs dessins.

\section{Résultats}

Juan

Juan est un garçon d'origine vénézuélienne âgé de 11 ans. Au moment de l'entrevue, il fréquente une classe d'accueil depuis 8 mois. II a immigré avec son frère, son beau-père et sa mère, qui avait déjà de la famille au Québec. Les premières démarches pour l'immigration ont débuté alors que Juan était encore bébé. Déjà au Venezuela, Juan disait à ses camarades de classe qu'il allait venir au Canada :

Et quand l'immigration, moi je me rappelle que chaque année, de la première année, je disais à mes amis "Hey, moi je vais aller à Canada", chaque année, chaque année, et jusqu'à $5^{\mathrm{e}}$ année ils m’ont dit "C'est pas vrai..."[sic] 
Le projet migratoire n'était donc pas nouveau pour Juan. II est aussi intéressant de noter que sa maman est née au Venezuela de parents colombiens, eux-mêmes enfants d'immigrants. Comme elle le souligne « Por eso, la palabra inmigración para nosotros es muy conocida [sic] " [C'est pour cela que le terme immigration est très familier pour nous]. Bien qu'à la maison les coutumes, la nourriture et les objets (ex. drapeau) soient vénézuéliens, la mère mentionne que les identités vénézuélienne et colombienne "es mélangé [sic] ». À ce sujet, Juan navigue entre les deux, surtout sur le plan de la langue, quand il utilise des expressions typiquement colombiennes avec des Colombiens et retourne à l'espagnol vénézuélien avec des Vénézuéliens.

À l'école, Juan est un jeune garçon qui aime apprendre, surtout les autres langues. Le fait de ne pas maîtriser aussi bien le français fait que, selon lui, il est plus réservé et n'est pas tout à fait lui-même :

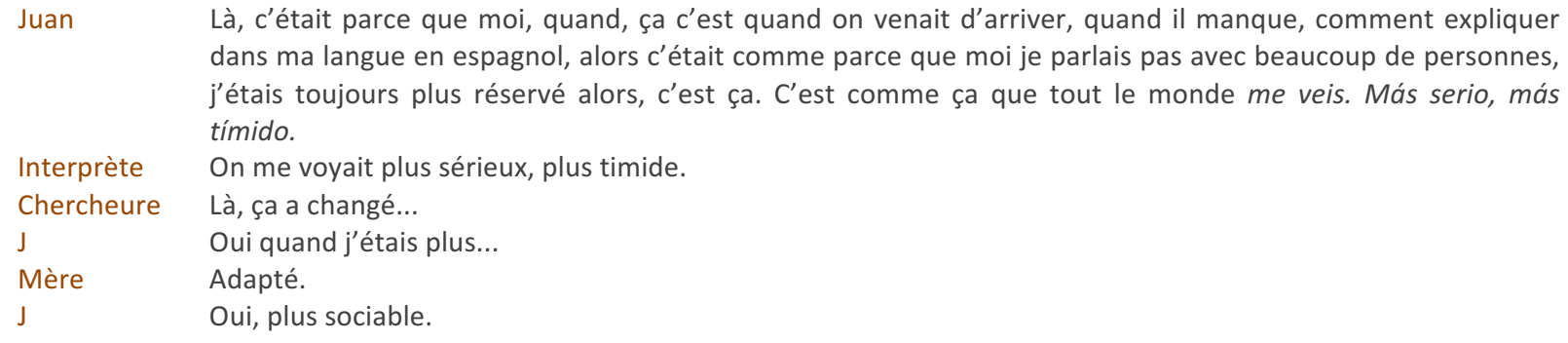
dans ma langue en espagnol, alors c'était comme parce que moi je parlais pas avec beaucoup de personnes, j'étais toujours plus réservé alors, c'est ça. C'est comme ça que tout le monde me veis. Más serio, más tímido.

Interprète On me voyait plus sérieux, plus timide.

Chercheure Là, ça a changé...

J Oui quand j'étais plus...

Mère Adapté.

J Oui, plus sociable.

La langue semble aussi être un enjeu pour son enseignante qui mentionne que Juan est conscient de l'obstacle que peut être son accent pour être compris des autres :

Donc, et Juan lui il est bon, il s'est rendu compte que quand j'enseigne, quand je suis en avant, mon accent, les autres ne comprennent pas nécessairement, ils se rendent compte eux-mêmes de leurs lacunes et ils travaillent dessus parce qu'ils fournissent [sic] devant quelqu'un.

La question de la langue revient dans plusieurs dessins qu'il réalise lors des ateliers et ce thème est généralement associé à celui de l'étrangeté. Dans la figure 1, Juan dessine un " mélange d'animaux qui parle plusieurs langues » (il écrit " je parle » en arabe, en espagnol et dans une langue étrangère, suivi de " $L O L$ »). ॥ dessine également un personnage en dessous « qui se cache parce qu'il était bizarre et qu'il a peur [sic] ».

\section{Figure 1 : Un mélange bizarre d’animaux}

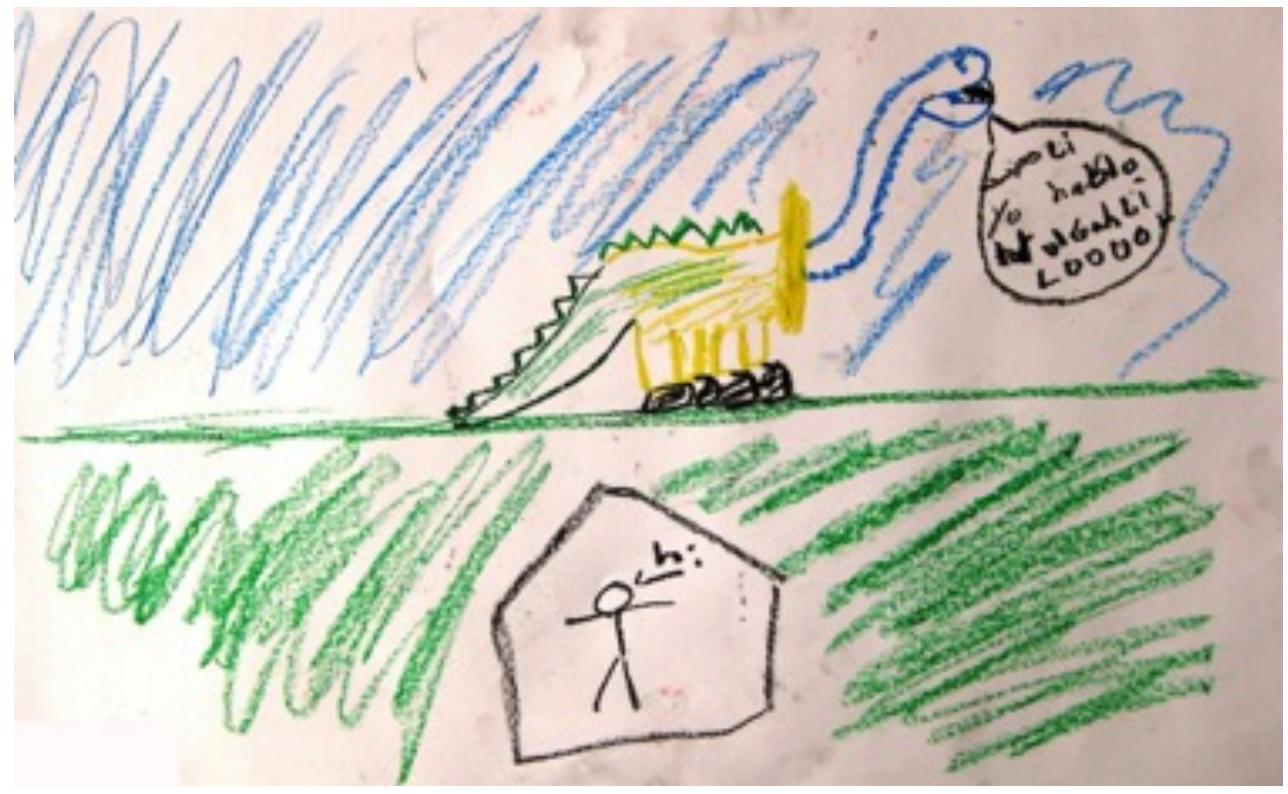


Dans un autre dessin (Figure 2), il représente « quelqu'un qui était bizarre dans son village, parce qu'il a une main à 6 doigts, et qui cache sa bouche [sic] ". Le personnage, aux yeux de couleurs différentes, se trouve entre deux maisons. La mère fait remarquer que ce personnage ressemble à un personnage de manga et, après vérification, ce personnage est en effet grandement inspiré du ninja Kakashi dans le manga Naruto (Kishimoto, 2002). Encore une fois, Juan fait parler le personnage en plusieurs langues, cette fois-ci en anglais et en français, et celui-ci dit « $h i$ je suis bizzarre [sic] ». Tout comme dans la figure 1, le thème de l'étrangeté est ici associé à celui de la mixité. Cependant, un certain sentiment d'étrangeté peut également être ressenti envers ce qui est différent, comme le démontre le cas de Safia dans la section qui suit.

Figure 2 : Quelqu'un de bizarre qui cache sa bouche

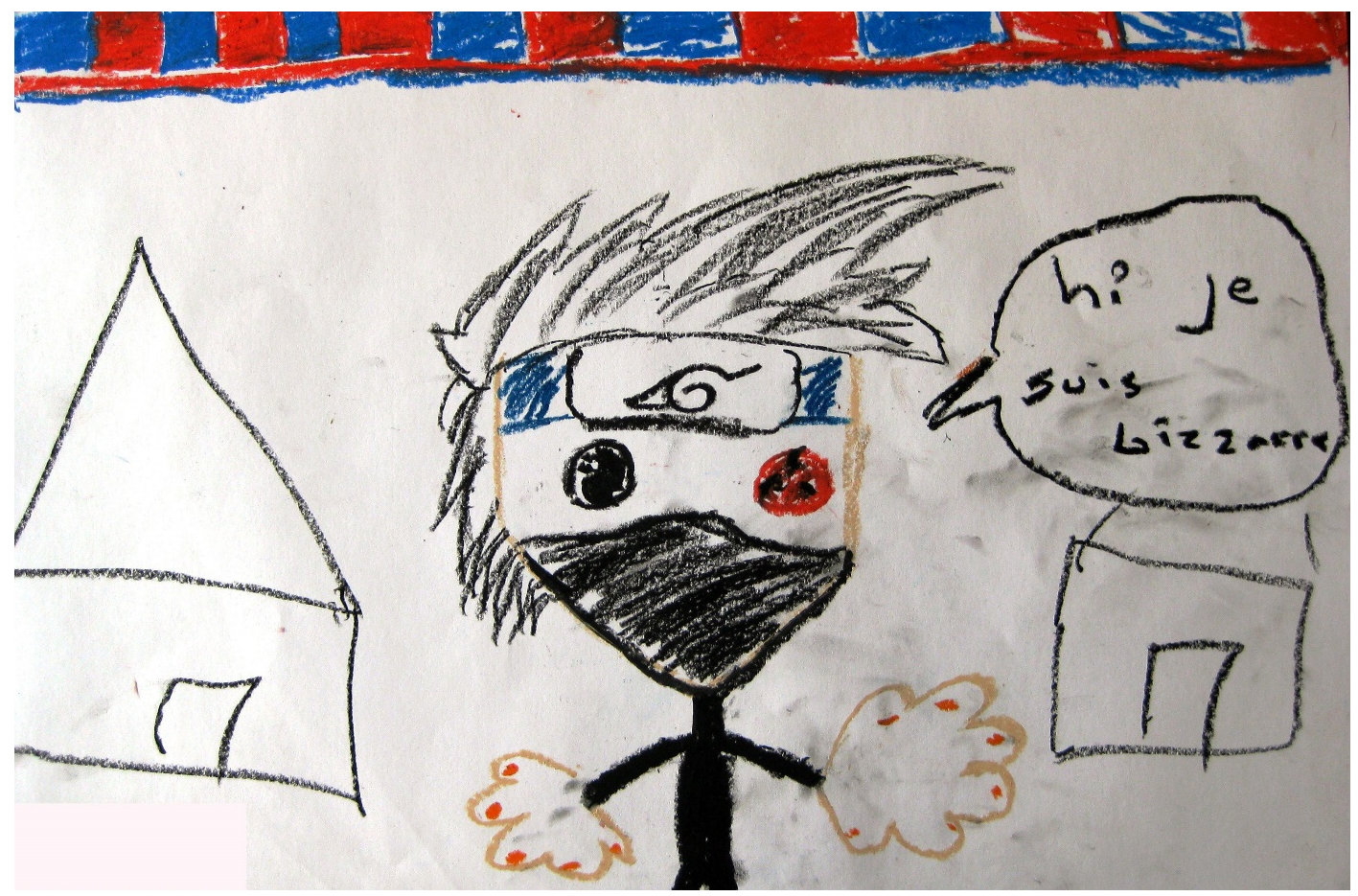

Safia

Safia est dans la même classe que Juan et elle est aussi âgée de 11 ans. Elle est née, quant à elle, en Égypte. Pour la famille, l'immigration en soi s'est bien déroulée, mais les parents ont fait beaucoup de sacrifices en venant s'installer au Québec, quittant des emplois prestigieux et bien rémunérés. Au moment de l'entrevue, les parents sont en processus d'obtenir les acquis nécessaires afin de pouvoir intégrer des ordres professionnels québécois correspondant à leurs qualifications.

Safia est très contente de participer à l'entrevue et elle en profite pour parler de son pays d'origine et de ce qu'elle a quitté. Tout comme ses parents, elle parle de l'Égypte avec émotion. Elle aime beaucoup partager sur la culture égyptienne et sur la langue arabe, dont elle montre les rudiments à ses camarades de classe ainsi qu'à la chercheure (écrire les chiffres). Cet attachement à son pays d'origine est aussi présent dans ses dessins et les commentaires qu'elle fait à leur sujet. En effet, le dessin qu'elle aime le plus est le drapeau de l'Égypte à l'endos duquel elle a dessiné un paysage vert qui lui rappelle les vallées fertiles du Nil. Safia est amie avec d'autres filles de sa classe originaires du Moyen-Orient, dont l'une est arabophone yéménite. Elle parle à plusieurs reprises de cette jeune fille et la dessine également. Dans la figure 3, on peut la voir aux côtés d'un extra-terrestre que Safia présente comme étant un garçon de sa classe d'une autre origine sociolinguistique. 
Figure 3 : Ce qui ne me ressemble pas est étrange

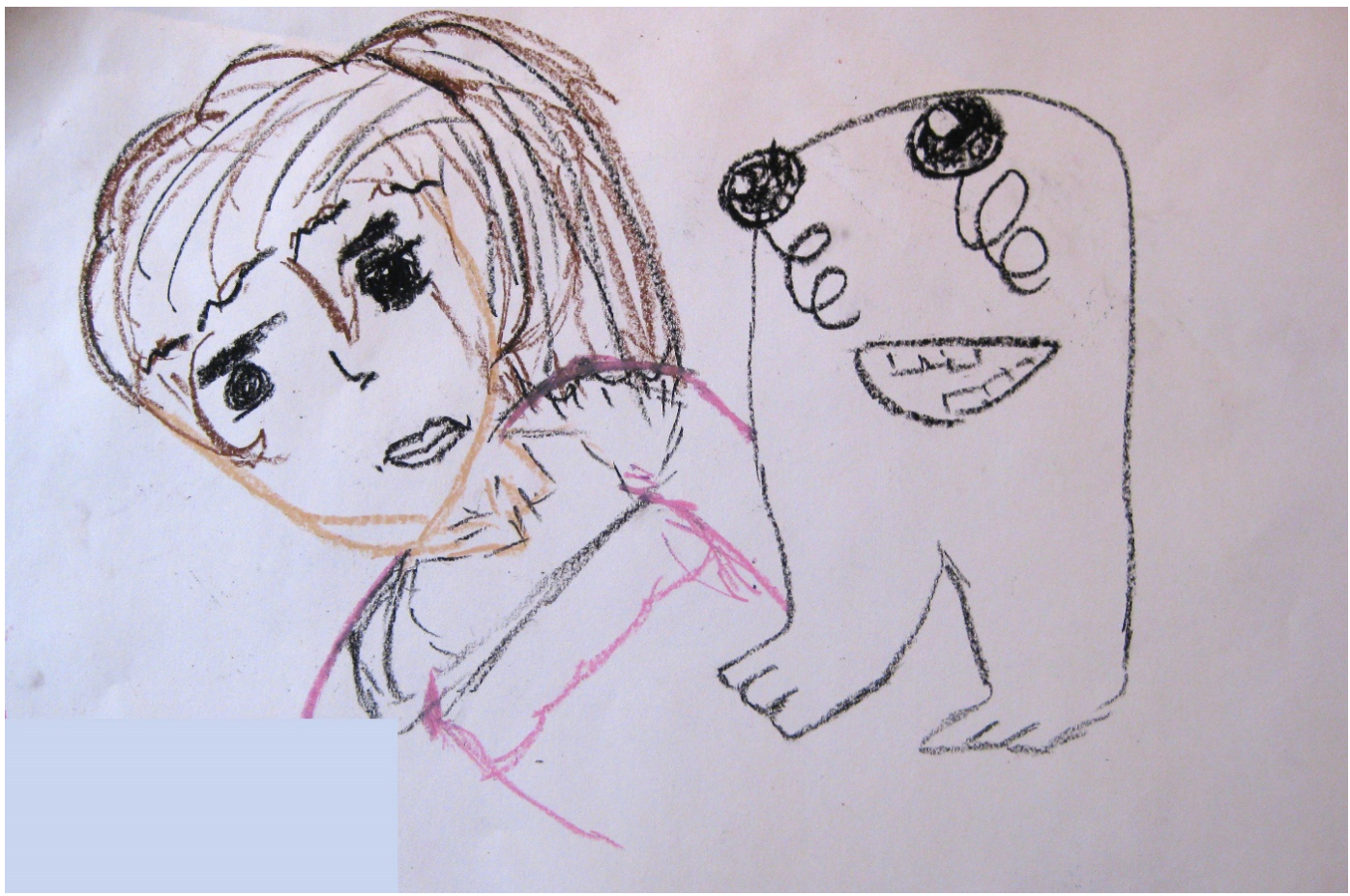

Au fil des ateliers, Safia dessine de plus en plus ce qu'elle associe à des mangas. Pendant l'entrevue, elle me présente le dessin de Yotsuba, personnage de manga (Figure 4).

Figure 4 : Une fille bizarre qui apprend sur le monde autour d'elle

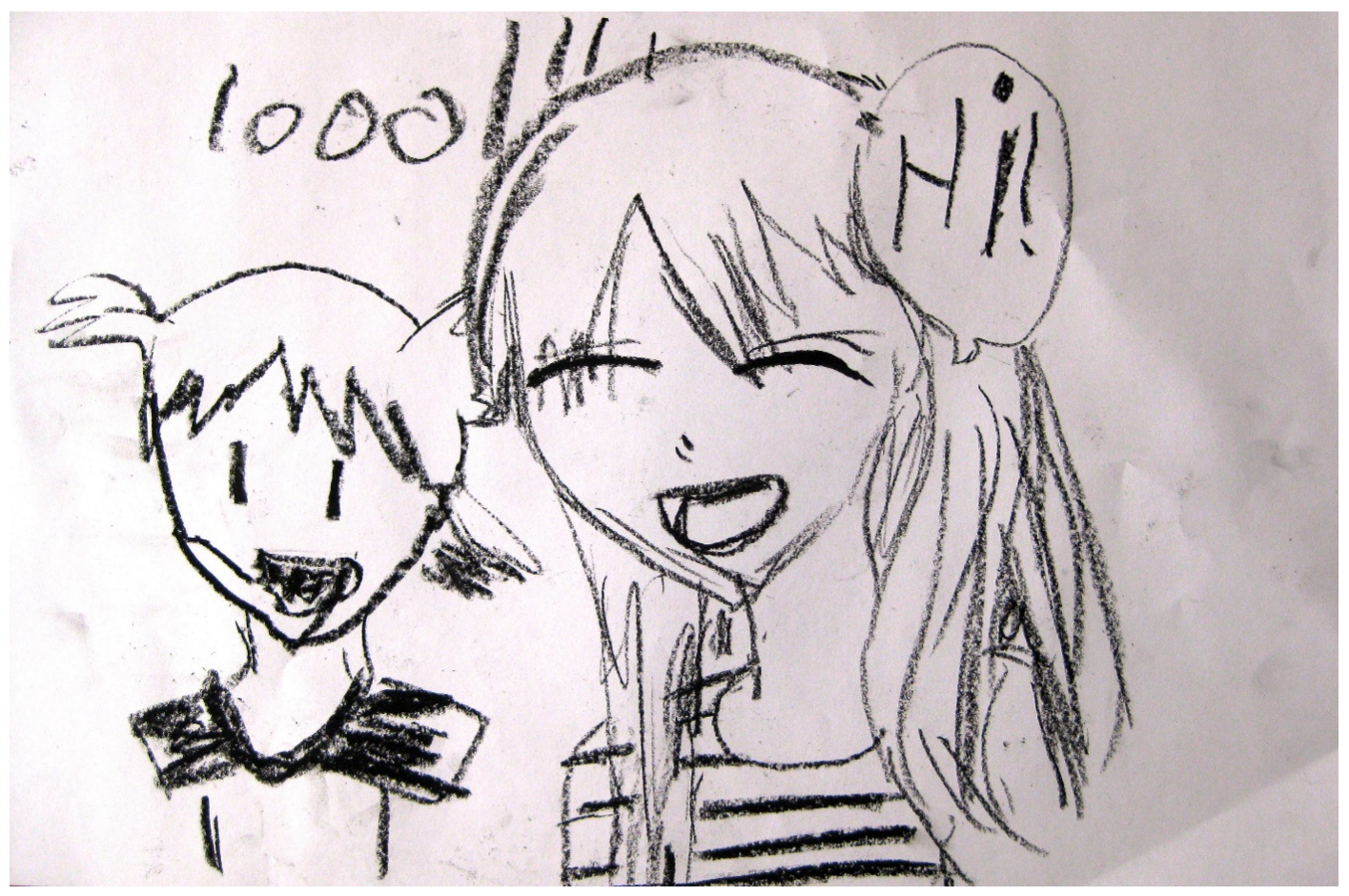


Safia aime bien ce livre. Elle y aime les émotions qu'elle trouve drôles :

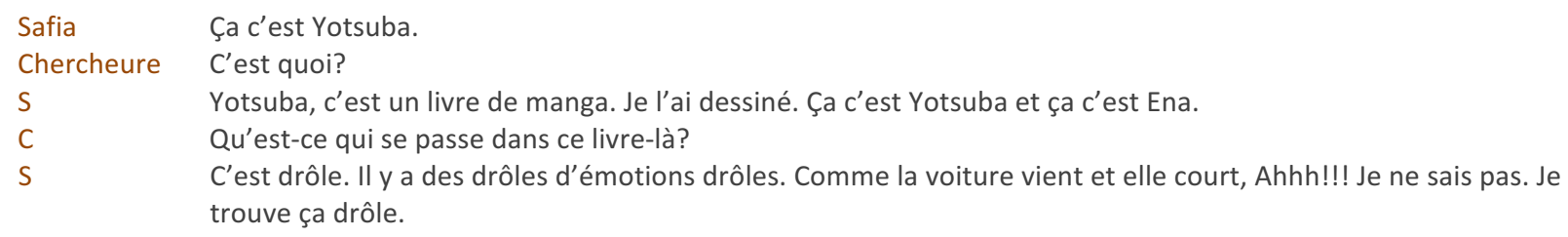

Cependant, le sens précis qu'elle accorde au mot " drôle » est nébuleux. Est-ce plutôt dans le sens de " comique » ou bien de "bizarre "? Dans ses dessins de mangas, comme c'est le cas avec celui de Yotsuba, Safia utilise également des expressions anglophones ou associées à la culture des jeunes telles " $L O L$ ", "XD », qu'elle doit expliquer aux adultes présents :

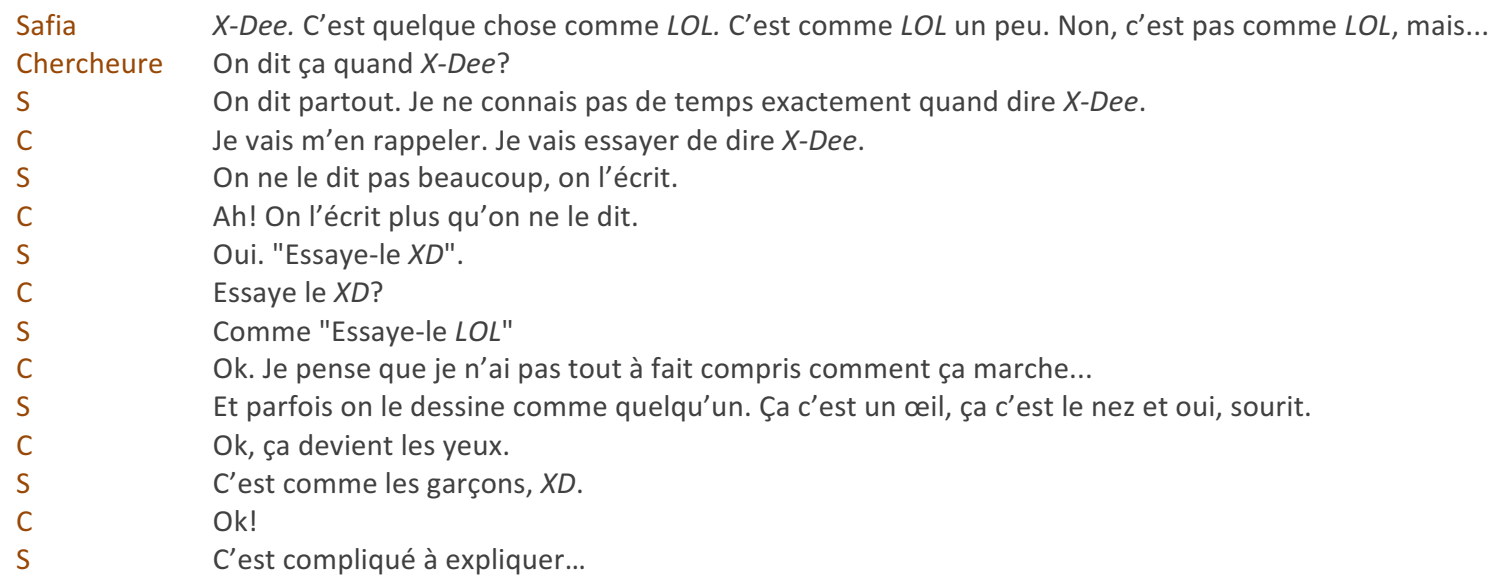

La jeune fille utilise un langage propre à la culture des jeunes qu'elle tente d'expliquer, sans grand succès, aux adultes. Ce langage semble contribuer au mouvement de ses frontières identitaires, en s'éloignant des personnes qui ne possèdent pas les mêmes codes linguistiques qu'elle.

\section{Être étrange...}

Dans les dessins de Juan et de Safia, le mouvement des frontières identitaires est symbolisé, entre autres, par les thèmes de l'étrangeté ou du bizarre et de la similarité à l'autre. Le thème de l'étrangeté est récurrent dans les dessins des deux jeunes, comme l'illustrent les figures 1 à 4. Cependant, les deux jeunes l'expriment et en font usage de manière distincte. Juan le fait de façon explicite dans le contenu des paroles exprimées par les personnages de ses dessins (figure 2) ou dans les commentaires qu'il fait à propos de ceux-ci (« un personnage qui se cache parce qu'il était bizarre ») (figure 1). Safia exprime l'étrangeté de façon plus subtile et implicite, tel que l'indiquent la présence de l'extraterrestre dans la figure 3 et le dessin de Yotsuba, petite fille bizarre (figure 4). Malgré une expression différente de l'étrangeté, les deux jeunes emploient des éléments graphiques et linguistiques similaires, soit les mangas et des expressions populaires se rapportant à la culture des jeunes.

Le dessin semble avoir permis à Juan d'exprimer à la fois son sentiment d'étrangeté et son désir d'être comme les autres. Tout d'abord, le thème de l'étrangeté est présent dans le dessin de la figure 1 par la présence d'un animal étrange composé de différents animaux et qui parle plusieurs langues. Pour Juan, ce sentiment d'être étrange pourrait relever du fait de posséder un héritage socioculturel et linguistique mixte (vénézuéliencolombien/québécois). Le lien entre étrangeté et mixité des langues est également présent dans le dessin de la figure 2, où le personnage dit clairement qu'il est "bizzarre [sic] ", et ce, en utilisant un mélange de français et d'anglais derrière une bouche cachée par un foulard. II est possible que Juan exprime une certaine conscience des difficultés que peut lui apporter son accent et que son enseignante mentionne aussi durant l'entrevue. Toujours dans ce dessin, il semble également exister un lien entre étrangeté, dissimilarité et position d'entre-deux. La 
différence vis-à-vis de l'autre semble altérer l'intégrité identitaire de Juan, son ipséité (Jodelet, 2008; Ricœur, 1990). En effet, le sentiment d'être bizarre, voire anormal, s'exprime à travers la représentation graphique d'un personnage de manga qui se situe entre deux maisons, possède des yeux de couleurs différentes et des mains au nombre de doigts inégal. II y a également une bande en haut du dessin qui présente les couleurs rouge et bleu en alternance, rappelant la possibilité de choisir entre deux options identitaires principales : vénézuélien-colombien et québécois. Cependant, cette situation d'entre-deux ne semble pas nécessairement confortable pour Juan. Lors d'un retour sur cette image, il affirme que c'est un dessin sur la discrimination, les gens riant du personnage parce qu'il est bizarre. Cette situation d'entre-deux pourrait faire référence à la revendication d'une identité québécoise non reconnue, à la fois dans le passé, alors que Juan pouvait déjà être considéré " étrange » dans son propre pays, du fait qu'il exprimait de façon récurrente son départ prochain pour le Canada, à la fois dans le présent, alors que la société d'accueil ne le considère possiblement pas encore comme québécois. Pour Juan, cette situation semble avoir trouvé écho dans l'histoire du manga Naruto, qui aborde effectivement la quête d'une identité véritable (Rankin-Brown et Brown Jr., 2012).

En ce qui concerne Safia, elle utilise des mangas pour exprimer un certain sentiment d'étrangeté de manière plus implicite. Dans la figure 4, on remarque deux personnages de manga, Yotsuba et sa sœur Ena, souriant et riant, comme le souligne l'expression « LOOOL ». En apparence, tout va bien. Cependant, l'expression d'étrangeté réside plutôt dans le choix particulier de cette histoire de manga, qui relate les expériences quotidiennes d'une petite fille adoptée apprenant sur le monde autour d'elle et commettant plusieurs impairs à ce sujet (Azuma, 2009). Au fil de I'histoire, Yotsuba découvre le mode de fonctionnement de son environnement, un peu à la manière d'une enfant immigrante. Safia pourrait donc s'identifier à l'expérience de Yotsuba et à une identité "étrange ", adjectif qui s'apparente au mot « étranger » et qui fait référence à la non-appartenance à la nation où l'on vit.

Le sentiment d'aliénation est plus présent dans un autre dessin qu'a réalisé Safia. Contrairement à Juan qui semble s'identifier aux personnages bizarres, c'est plutôt l'autre, qui est différent de soi, qui est dépeint comme l'étranger dans le dessin de la figure 3. Dans cette image, une jeune fille de qui Safia est proche et avec qui elle partage des codes communs (ex. la langue), est dépeinte sous forme humaine alors qu'un garçon de leur classe, ayant un bagage sociolinguistique différent, est représenté sous forme d'extraterrestre. La représentation de l'autre sous forme d'un extraterrestre met en lumière la comparaison à elle-même qu'effectue Safia et semble rendre compte, par projection, de l'altérité vécue (Jodelet, 2008). Dans ce cas-ci, la comparaison se traduit par l'usage d'une stratégie de différenciation, afin que la jeune fille puisse déplacer le plus loin possible les frontières de son identité (Marc, 2005). Cependant, elle ne semble pas nécessairement bien dans cette situation puisque le regard de la jeune fille du dessin paraît triste et nostalgique et que celle-ci pourrait donner l'impression d'être attachée. Safia pourrait aussi projeter son sentiment d'étrangeté sur un de ses camarades de classe, ce qu'elle exprime dans ce dessin.

\section{... à la recherche d'universalité}

Les mangas ont beaucoup gagné en popularité auprès des jeunes ces dernières années (Schwartz et RubinsteinÃvila, 2006). Dans le cadre des ateliers Art et Contes, plusieurs élèves reproduisent des dessins de manga qu'ils lisent et qu'ils échangent entre eux. Parfois, le dessin de manga se fait à partir d'une image qu'ils cherchent à répliquer, parfois non. Peu importe la manière dont les mangas sont introduits dans les dessins, ceux-ci semblent posséder une attraction particulière pour les jeunes immigrants préadolescents, qui y trouvent des repères pour s'y identifier. Les mangas présentent effectivement des stimuli ambigus et un métissage de différents codes pouvant être attrayants pour des jeunes de ce groupe d'âge (Schwartz et Rubinstein-Ãvila, 2006). En effet, les personnages rappellent des adolescents d'apparence physique occidentale, sans toutefois que l'âge, le genre et l'origine ethnoculturelle soient bien définis, rendant les frontières identitaires floues et indistinctes (Rankin-Brown et Brown Jr., 2012). Les mangas permettent alors de se reconnaître dans l'autre tout en réduisant ce sentiment d'être autre (Ricœur, 1990).

Le dessin, en particulier les mangas, permet à ces jeunes de reprendre un certain pouvoir sur ce qu'ils vivent et de se remettre d'une expérience d'aliénation, comme dans le cas de l'histoire du manga Naruto que représente Juan sur la figure 2 (Ito, 2011). En effet, les lecteurs de manga doivent être capables de naviguer entre différentes 
modalités de lecture pour être compétents. Un manga est composé d'encadrés d'images et de textes pouvant être lus dans différentes directions: de droite à gauche, de gauche à droite, ou parfois de haut en bas. Les lecteurs doivent également naviguer entre différentes langues, puisque les mangas peuvent être composés de dialogues écrits en scripts romains, japonais ou chinois (Schwartz et Rubinstein-Ãvila, 2006). Par ailleurs, la réalisation de manga requiert certaines connaissances relatives à la "grammaire " graphique du manga, puisqu'il existe des étapes prédéfinies pour réaliser le dessin ainsi que des consignes quant à la manière de représenter les personnages et les émotions (Cohn, 2010). Maîtriser le dessin de manga permet alors le passage d'un sentiment d'étrangeté à un sentiment de compétence chez des jeunes immigrants susceptibles de vivre un sentiment d'aliénation, en même temps qu'elle permet de faire le pont entre des jeunes de cultures différentes qui partagent les connaissances et les compétences nécessaires à la réalisation de ceux-ci. La maîtrise de ce type de dessin, populaire chez les jeunes, leur permet de s'identifier à une culture commune alors qu'ils sont différents les uns des autres. Comme le souligne Ricœur (1990), l'identification " de l'autre entre dans la composition du même » (p. 143) : en s'identifiant à autrui et en intériorisant certaines de ses caractéristiques, l'altérité est pour ainsi dire annihilée.

D'autres marqueurs permettent également d'unir les jeunes dans une culture commune: les expressions populaires issues d'un langage propre à leur génération, telles $X D, L O L$ ou l'utilisation de l'anglais. À titre d'exemple, Safia passe un bon moment lors de l'entrevue à expliquer ce que veut dire "XD", ou à définir l'expression « être folle » qu'elle utilise pour caractériser la jeune fille de la figure 3 :

Safia Non, non, c'est pas vraiment folle. Drôle.

Chercheure Ok! Drôle! Je comprends.

S Moi quand je parle avec mes amis, c'est comme ça, ils comprennent, mais les adultes ne comprennent pas les expressions...

Dans ce court extrait d'entrevue, Safia met l'accent sur l'altérité en exprimant clairement la séparation existant entre le monde des adultes et celui des jeunes, et ce, avec l'exemple des expressions que les jeunes utilisent. Bien que les conflits intergénérationnels puissent être fréquents à la préadolescence (Sonja, 2016), il est possible qu'afin de rétablir un sentiment d'ipséité dans son identité et d'atténuer le sentiment d'altérité (Jodelet, 2008), Safia ait cherché à ressentir les bienfaits d'un sentiment d'appartenance à sa classe, tous étant concernés par la culture des jeunes. L'universalité pourrait ainsi empêcher l'altérité de se manifester (Ricœur, 1990). Pour Safia, la recherche d'universalité se traduit par l'usage de stratégies d'assimilation pour se rapprocher des autres jeunes, en mesure de la comprendre puisqu'ils partagent un héritage commun (Marc, 2005). Cette recherche d'universalité provoque cependant la création d'un sentiment d'altérité envers les adultes, ce qui transparaît par le recours à des stratégies de différenciation vis-à-vis d'eux (qui ne comprennent pas les expressions populaires des jeunes). Dans le cas de Juan, ces expressions populaires sont beaucoup moins fréquentes dans ses dessins et Juan ne les commente pas lors de l'entrevue, contrairement à Safia. II semble les utiliser sans qu'elles revêtent une importance particulière pour lui ou sans qu'elles constituent une barrière entre le monde des jeunes et celui des adultes. Safia et Juan utilisent donc différemment les mangas et les expressions populaires afin de concrétiser le mouvement des frontières identitaires entre eux et les autres.

\section{Conclusion : trouver l'équilibre}

Les jeunes rencontrés lors de cette étude expriment leur sentiment et leurs perceptions de l'altérité dans les images créées dans le cadre d'ateliers d'expression créatrice en milieu scolaire. Par le dessin, ils négocient de façon complexe l'équilibre entre l'expression de l'étrangeté et celle de la ressemblance à l'autre, ce qui semble leur permettre de conserver un certain sentiment d'ipséité. En effet, l'exploration de l'altérité par le dessin semble leur avoir permis de donner sens à leur expérience d'immigration et de se (re)construire une identité signifiante. Comme le souligne Jodelet (2008), " "l'autre-semblable" [est] le médiateur de l'identité, de la représentation et de la connaissance en ce qu'il complète, réfléchit et donne sens à ce que le sujet éprouve " (p. 15). Ainsi, la construction de leur identité passe nécessairement par la perception de l'altérité et, pour ce faire, les jeunes immigrants ont eu recours à des stratégies d'assimilation et de différenciation qui ont déplacé les frontières de leur(s) identité(s). Ces stratégies sont visibles dans leurs dessins par l'utilisation de marqueurs identitaires qui leur permettent de trouver cet équilibre en leur fournissant une façon de négocier des identités alternatives. Cela se 
traduit, entre autres, par l'utilisation du manga et d'expressions populaires, qui permettent à la fois d'exprimer un sentiment d'étrangeté et de le transformer en pouvoir. À cet effet, le dessin semble soutenir de façon positive le processus de transformation identitaire.

\section{Références bibliographiques}

Anzieu, A., Barbey, L., Bernard-Nez, J., et Daymas, S. (2012). Le travail du dessin en psychothérapie de l'enfant. Paris : Dunod.

Armand, F. (2012). L'intégration linguistique, scolaire et sociale des élèves allophones d'origine immigrante dans la région du Grand Montréal : portrait des modèles et perceptions des acteurs. Montréal : CEETUM (Centre d'études ethniques des universités montréalaises). http://www.ceetum.umontreal.ca/documents/capsules/2012/integration-linguistique-2012.pdf

Augé, M. (1994). Pour une anthropologie des mondes contemporains. Paris : Éditions Aubier.

Azuma, K. (2009). Yotsuba\&! (vol. 1). New York : Yen Press.

Barth, F. (1995). Les groupes ethniques et leurs frontières. Dans P. Poutignat et J. Streiff-Fenart (dir.), Théories de l'ethnicité (p. 203-249). Paris : Presses universitaires de France.

Camilleri, C., Kastersztein, J., Lipiansky, E. M., Malewska-Peyre, H., Taboada-Leonetti, I., et Vasquez, A. (1990). Stratégies identitaires. Paris : Presses universitaires de France.

Cohn, N. (2010). Japanese visual language: The structure of manga. Dans T. Johnson-Woods (dir.), Manga: An Anthology of Global and Cultural Perspectives (p. 187-203). New York: The Continuum International Publishing Group.

De Koninck, Z., et Armand, F. (2011). Le choix des modèles de service offerts aux élèves issus de l'immigration au Québec : entre réalisme, tradition et innovation. Canadian Issues, 29-34.

Devereux, G. (1985). Ethnopsychanalyse complémentariste. Paris : Flammarion.

Devereux, G., La Barre, W. et Sinaceur, H. (2012). De l'angoisse à la méthode dans les sciences du comportement. Paris : Flammarion.

Dosse, F. (2006). L'importance de l'œuvre de Paul Ricœur pour la pratique historienne. Bulletin de la Société de L'histoire du protestantisme français, 152(4), 647-666.

Équipe de recherche et d'intervention transculturelles (Erit). (2010). Art et Contes. Manuel de formation. Ateliers d'expression créatrice. Montréal : Erit.

Faircloth, B. S. (2012). « Wearing a mask » vs. connecting identity with learning. Contemporary Educational Psychology, 37(3), 186-194.

Fouquet, T. (2007). Imaginaires migratoires et expériences multiples de l'altérité : une dialectique actuelle du proche et du lointain. Autrepart, (41), 83-98.

Frie, R. (2011). Identity, narrative, and lived experience after postmodernity: Between multiplicity and continuity. Journal of Phenomenological Psychology, 42(1), 46-60.

Gouvernement du Canada et Immigration Canada. (2015). Faits et chiffres 2014 - Aperçu de l'immigration : Résidents permanents. https://ouvert.canada.ca/data/fr/dataset/2fbb56bd-eae7-4582-af7d-a197d185fc93 (consulté 9 juin 2016)

Gouvernement du Québec (1988). Loi sur l'instruction publique, RLRQ c I-13.3 § (1988) [Internet]. http://www2.publicationsduquebec.gouv.qc.ca/dynamicSearch/telecharge.php?type=2\&file=/I_13_3/I13_3. html 
Gouvernement du Québec, Ministère de l'Immigration, de la Diversité et de l'Inclusion. (2015). Fiche synthèse sur I'immigration et la diversité ethnoculturelle au Québec. Québec : Gouvernemente du Québec. http://www.midi.gouv.qc.ca/publications/fr/recherches-statistiques/FICHE_syn_an2014.pdf

Hawkins, B. (2002). Children's drawing, self expression, identity and the imagination. International Journal of Art and Design Education, 21(3), 209-219.

Ito, G. (2011). Particularities of boys' manga in the early 21st century: How NARUTO differs from DRAGON BALL. Dans J. Berndt (dir.), Intercultural crossovers, transcultural flows: Manga/Comics. Global manga studies vol. 2 (p. 9-16). Kyoto : International Manga Research Center, Kyoto Seika University. http://imrc.jp/images/upload/lecture/data/Web_Version_Cologne\%E8\%AB\%96\%E9\%9B\%86.pdf

Jodelet, D. (2008). Formes et figures de l'altérité [Internet]. http://classiques.uqac.ca/contemporains/jodelet_denise/forme_figure_alterite/forme_figure_alterite.pdf

Kishimoto, M. (2002). Naruto, tome 1 (4 éd.). Bruxelles : Kana.

Marc, E. (2005). Psychologie de l'identité: Soi et le groupe. Paris : Dunod.

Ministère de l'Éducation, du Loisir et du Sport du Québec. (2014). Intégration linguistique, sociale et scolaire. Dans Programme de formation de l'école québécoise, enseignement primaire (p. 1-90). Québec : Gouvernement du Québec.

Phinney, J. S., et Ong, A. D. (2007). Ethnic identity development in immigrant families. Dans K. Deater-Deckard, M. H. Bornstein et J. E. Lansford (dir.), Immigrant families in contemporary society (p. 51-68). New York : The Guilford Press.

Pontalis, J.-B., et Laplanche, J. (2004). Vocabulaire de la psychanalyse ( $3^{\mathrm{e}}$ éd.). Paris : Presses universitaires de France.

Rankin-Brown, M., et Brown Jr., M. (2012). From samurai to manga: The function of manga to shape and reflect Japanese identity. Japan Studies Review, 16, 75-92.

Ricœur, P. (1990). Soi-même comme un autre. Paris : Seuil.

Schwartz, A., et Rubinstein-Ãvila, E. (2006). Understanding the manga hype: Uncovering the multimodality of comic-book literacies. Journal of Adolescent and Adult Literacy, 50(1), 40-49.

Sévigny, D. (2016). Portrait socioculturel des élèves inscrits dans les écoles publiques de l'île de Montréal: Inscriptions au 4 novembre 2015. Montréal : Comité de gestion de la taxe scolaire de l'île de Montréal.

Sinai, M., Kaplan, A. et Flum, H. (2012). Promoting identity exploration within the school curriculum: A designbased study in a Junior High literature lesson in Israel. Contemporary Educational Psychology, 37(3), 195205.

Song, M. (2003). Choosing ethnic identity. Cambridge : Polity Press.

Sonja, K. Č. (2016). Parent-adolescent conflict style and conflict outcome: Age and gender differences. Psihologija, 49(3), 245-262.

Stepakoff, S. (2007). The healing power of symbolization in the aftermath of massive war atrocities: Examples from Liberian and Sierra Leonean survivors. Journal of Humanistic Psychology, 47, 400-412.

Tap, P. (1988). La société Pygmalion? Intégration sociale et socialisation de la personne. Paris : Dunod.

Vinsonneau, G. (2002). L'identité culturelle. Paris : Armand Colin.

Vinsonneau, G. (2012). Dynamiques interculturelles et devenir des identités. Dans Mondialisation et identité culturelle (p. 73-118). Bruxelles : De Boeck Supérieur. 